EGU2020-5056

EGU General Assembly 2020

(c) Author(s) 2020. This work is distributed under

the Creative Commons Attribution 4.0 License.

\title{
Overview of the IBER-STILLING project: Assessment and attribution of wind speed and wind gust variability
}

\author{
Cesar Azorin-Molina ${ }^{1,2}$, Manola Brunet ${ }^{3}$, Enric Aguilar ${ }^{3}$, Jose A. Guijarro ${ }^{4}$, Amir A. Safaei Pirooz ${ }^{5}$, \\ Richard G.J. Flay ${ }^{5}$, Lorenzo Minola ${ }^{2}$, Gangfeng Zhang ${ }^{2,6,7}$, Joan-Albert Lopez-Bustins ${ }^{8}$, Tim R. \\ McVicar $^{9,10}$, and Deliang Chen ${ }^{2}$ \\ ${ }^{1}$ Spanish National Research Council (CSIC), Centro de Investigaciones sobre Desertificacion, Moncada (Valencia), Spain \\ (cesar.azorin@uv.es) \\ ${ }^{2}$ Regional Climate Group, Department of Earth Sciences, University of Gothenburg, Gothenburg, Sweden \\ ${ }^{3}$ University Rovira i Virgili, Tarragona, Spain \\ ${ }^{4}$ State Meteorological Agency, Balearic Islands Office, Palma de Mallorca, Spain \\ ${ }^{5}$ Department of Mechanical Engineering, The University of Auckland, Auckland, New Zealand \\ ${ }^{6}$ State Key Laboratory of Earth Surface Processes and Resource Ecology, Beijing Normal University, Beijing, China \\ ${ }^{7}$ Academy of Disaster Reduction and Emergency Management, Ministry of Civil Affairs and Ministry of Education, Beijing \\ Normal University, Beijing, China \\ ${ }^{8}$ Climatology Group, Department of Geography, University of Barcelona, Barcelona, Spain \\ ${ }^{9} \mathrm{CSIRO}$ Land and Water, Canberra, ACT, Australia \\ ${ }^{10}$ Australian Research Council Centre of Excellence for Climate Extremes, Canberra, ACT, Australia
}

In a context of global climate change, the scientific community has evidenced a significant decrease in wind speed, a phenomenon known as «stilling». This climate trend has mainly been observed over mid-latitude continental surfaces since the 1980s. On the contrary, other studies have detected an increase in wind speed over ocean surfaces; and there is little conclusive scientific evidence on trends in wind speed across the troposphere. Furthermore, a reversal in global terrestrial stilling has recently been documented in few regional and global studies since the 2010s. The causes associated with the climate variability of wind speed have not yet been resolved and there are many uncertainties behind the «stilling» and «recovery» phenomenon because neither the quantity nor the quality of wind speed observations is adequate. This contribution shows an overview of the IBER-STILLING project (RTI2018-095749-A-I00) funded by the Spanish Ministry of Science, Innovation and Universities. This project aims to move forward on the assessment of wind speed and wind gusts variability and underlying causes globally, with emphasis on the Spanish territory and surrounding ocean (Atlantic) and sea (Mediterranean) surfaces. The IBER-STILLING project will collect and generate climate information of wind speed from different data sources; climate data will be subject to a comprehensive protocol for quality control and homogenization. The statistical analysis of these climate databases will allow characterizing trends and climatic cycles of wind speed, allowing a pioneering global analysis of wind speed over continental and ocean surfaces, and across the boundary layer and the entire troposphere. The project will also conduct wind-tunnel experiments to quantify biases introduced by anemometers devices. 
